\title{
Introduction: 3D imaging in lung biology
}

\author{
Christian Mühlfeld ${ }^{1,2} \cdot$ Douglas J. Taatjes ${ }^{3,4}$
}

Published online: 11 February 2021

(c) The Author(s), under exclusive licence to Springer-Verlag GmbH, DE part of Springer Nature 2021

The genesis for this Special Issue devoted to "3D Imaging in Lung Biology" emerged when the two of us met for the first time as faculty at the Jackson Laboratory's course, "Phenotyping Mouse Models of Human Lung Disease", in October 2019. Discussing lung stereology and other 3D imaging considerations, while at the same time, leveraging our editorial affiliation with Histochemistry and Cell Biology, we came upon the idea of developing a Special Issue of the journal devoted to this topic.

One of the major and inherently esthetic principles in biological sciences is the close relationship between the structures composing an organ and its/their function. In the lung, this relationship is particularly close and therefore, imaging of the lung not only reveals its structural composition but also reveals its functional status during development and under normal and pathological conditions. The history of the discovery of the structure-function relationships of the lung is also a history of the development of microscopic techniques: before the electron microscopic (EM) era had begun, it was unclear how the interface between air and blood was composed, whether the capillaries were "naked" or covered by a fragmented or continuous epithelium. The reason for this uncertainty was the functionally relevant, extremely thin barrier between air and blood and the impossibility to resolve it by light microscopy (LM). The invention of transmission electron microscopy (TEM) made it possible to resolve the layers of the air-blood barrier and solve this

Douglas J. Taatjes

douglas.taatjes@med.uvm.edu

1 Institute of Functional and Applied Anatomy, Hannover Medical School, Member of the German Center for Lung Research (DZL), Hannover, Germany

2 Biomedical Research in Endstage and Obstructive Lung Disease Hannover (BREATH), Member of the German Center for Lung Research (DZL), Hannover, Germany

3 Department of Pathology and Laboratory Medicine, University of Vermont, Burlington, VT 05405, USA

4 Microscopy Imaging Center, Larner College of Medicine, University of Vermont, Burlington, VT 05405, USA question unequivocally (Low 1953). Apparently, the thinness of the air-blood barrier can only be achieved by reducing the amount of cellular contents (e.g., nucleus, mitochondria) that would increase the thickness at the direct contact sites between capillary endothelium and alveolar epithelium (for a review, see Maina and West 2005). This functional adaptation results in another peculiarity which was difficult (but not impossible) to answer by TEM, the question of the non-nucleated plates: parts of the alveolar epithelium do not seem to be connected to a nucleus-containing part of the epithelium which could be explained by extensions of the type I epithelial cells projecting from one side of the alveolar septum to the other (Weibel 1971). The 1960s and 1970s were the golden era of TEM-based lung research which was dominated by Ewald Weibel at the University of Bern, Switzerland. Another important discovery was the morphological characterization of the surface tension-lowering intra-alveolar surfactant as an extremely thin layer covering the alveolar epithelium at its top, made possible by the fixation of the lung by vascular perfusion (Weibel and Gil 1968; Gil and Weibel 1969). For a more detailed view on the microscopic history of the alveolar epithelium, please refer to Ochs et al. (2016).

For relating structures to their function, it is often not enough to describe them qualitatively but to express them in quantitative terms. As such, microscopists have always been tempted to "count" the number of the objects of interest. The two-dimensionality of section-based microscopy, however, may not only lead to misinterpretation of the true three-dimensional nature of an object but also carries a great source of bias in the quantification of objects (Mendis-Handagama 1992). The method of choice to overcome the problems associated with microscopic morphometry is design-based stereology (reviewed in detail in Hsia et al. 2010; Ochs and Mühlfeld 2013). Lung stereology is based on Ewald Weibel's pioneering work in the 1960s and 1970s (e.g., Weibel 1963; Gehr et al. 1978) which was brought to full power by the methodological developments by Hans Gundersen in the 1980s and 1990s (e.g., Gundersen 1986; Gundersen et al. 1988), particularly the disector technique 
(Sterio 1984) that was later applied to the number of alveoli (Ochs et al. 2004) and septal capillaries (Willführ et al. 2015).

However, the interest in microscopy- or morphologybased lung research began to decline in the 1980s due to the rise of a vast amount of molecular techniques enabling the acquisition of new types of knowledge. Another reason was the seemingly lack of the development of influential microscopic techniques. However, already at that time, important developments were made both at LM and at EM level. Confocal laser scanning microscopy (CLSM) and electron tomography (ET) marked the beginning of 3D investigation of objects based on thick sections (Cox et al. 1982; Shotton and White 1989; Taatjes et al. 2001; McEwen et al. 1986; Baumeister et al. 1999). Apparently, CLSM has often been misinterpreted as a more modern, fancier type of fluorescence microscopy based on thin 2D sections while its great advantage was the possibility to gain 3D knowledge from thick sections. Nevertheless, the further direction of microscopic development was clearly set to explore biological objects in three dimensions. There is a vast amount of techniques such as various types of super-resolution microscopy, including STED or STORM (Dyba and Hell 2003; Schermelleh et al. 2019), or volume EM, including serial block-face and focused ion beam scanning electron microscopy (e.g., Briggman and Bock 2012) whose full potential still needs to be applied to pulmonary research (Ochs et al. 2016).

The technological developments in the second major morphological field, namely radiology, are not less impressive, yet they are most often regarded independently of microscopy. Both imaging techniques, however, might solve the problems of the other when they are used in combination: radiological techniques are non-destructive, can be applied in the living state and provide truly 3D information, however, despite the great progress in micro- and nano-computed tomography, the resolution is still limited (Albers et al. 2018; Schittny 2018; Rawson et al. 2020). On the contrary, microscopic techniques require smaller objects of analysis to achieve their maximal resolution. It is time to combine and apply the new techniques and exploit their full potential in the investigation of the lung.

We are convinced that these are exciting times in morphology-based lung research, and that use of the newly available technology will lead to new exciting discoveries in the structure and function relationships of the lung in development, health and disease. To stimulate this process, the present special issue compiles a set of articles highlighting the possibilities of 3D image acquisition, analysis, and application in lung research.

Since rigorous and reproducible quantitative 3D imaging of any tissue or organ are underpinned by the principles of stereology, this Special Issue will begin with a state-of-the-art review of these principles focused on lung, by Knudsen et al. (2021). One of the truly outstanding features of stereological methods is that they can be applied to any $3 \mathrm{D}$ imaging data set, regardless of the instrumentation used for image acquisition. The review provides interesting historical background describing development of the techniques, as well as very informative images and tables explaining stereology-specific terminology and methodology. Finally, examples of stereological investigations of lung using 3D data sets acquired by electron microscopy, confocal microscopy, and X-ray-based instruments (such as $\mu \mathrm{CT}$ ) are described in detail, with examples provided in a summary table. Knudsen and colleagues (Krischer et al. 2021) then follow with an interesting original paper in which they utilized the stringent design-based ultrastructural stereological methods just introduced in their Review (Knudsen et al. 2021) to investigate the effects of ventilation pressure on the structure of the blood-gas barrier, ultrastructure of alveolar type II epithelial cells, and the intercellular surfactant pool in control and pre-injured rat lungs. Their detailed results, which could only have been obtained with rigorous adherence to stereological protocols, revealed that mechanical ventilation-induced ultrastructural alterations differ in control versus pre-injured lungs. Perez-Bravo et al. (2021) provide a most useful addition to the lung stereological literature concerning the initial tissue fixation protocol. They investigated the effects of employing differing airway pressure during inflation fixation on subsequent stereological parameters from neonatal mouse lungs. Comparing the results obtained from using three different airway pressures during fixation, they found that stereological features such as lung volume were indeed affected by the airway pressure, and thus recommend that a pressure of $20 \mathrm{cmH}_{2} \mathrm{O}$ should be used during inflation fixation of neonatal mouse lungs to prevent pressure-induced lung tissue alterations.

Borisova et al. (2021) describe a new X-ray tomographic method for achieving full-volume 3D reconstruction of an intact juvenile rat lung, providing imaging of the entire lung structure from the trachea to parenchyma in one data set in a temporal period minimizing tissue degradation artifacts. The manuscript provides detailed technical descriptions of the data acquisition process, together with data reconstruction and image stitching. Grothausmann et al. (2021) combined imaging of a whole rabbit lung with an X-ray source $(\mu \mathrm{CT})$, with serial section light microscopy imaging to provide a stereological analysis of pulmonary arterial branches. Extensive digital processing was applied to the 3D sets of images, including painstaking registration of the $\mu \mathrm{CT}$ data with the corresponding light microscopy images. This "proof-of-concept" study indeed demonstrated that the correlative use of these two imaging modalities, providing different degrees of resolution, can be used to assess the pulmonary vasculature in a quantitative manner. 
Schneider et al. (2021) provide a thorough and up-to-date review of the various imaging and analysis technologies to address volume electron microscopy of the lung. They cover in detail all of the electron microscopy techniques currently available for providing 3D details of lung ultrastructure, including serial sectioning TEM, electron tomography, serial block-face SEM, focused ion beam SEM, and array tomography. The review is beautifully illustrated, with informative figures and a table illustrating the principles and advantages and disadvantages of each technique. Finally, they describe post-processing and image analysis techniques especially useful when working with these large 3D data sets obtained from various lung samples. Mühlfeld et al. (2021) utilized two of the volume techniques described in the review just highlighted, namely focused ion beam SEM and electron tomography to investigate the function of the plate body, an organelle present in lung alveolar epithelial type II cells. Since plate bodies, speculated to be involved in surfactant protein A (SP-A) synthesis and trafficking have only been identified in certain mammalian species, they examined their 3D characteristics in archived lungs from goats and sheep. In conjunction with immunoelectron microscopy, their 3D volume analysis demonstrated that the plate body membrane is connected with rough endoplasmic reticulum, and though the plate membranes themselves were not immunolabeled for SP-A, a vesicular network component of the plate body was stained. Their results provided strong ultrastructural evidence that a vesicular component of the plate body is indeed involved in SP-A trafficking.

Salwig et al. (2021) present an original communication describing the application of light sheet microscopy for investigations of airway regeneration, focusing on epithelial Club cells in a lung injury model. They used an optimized solvent-based tissue clearing technique which preserved the mCherry fluorescence in their transgenic animals. Using the combined tissue clearing method together with light sheet microscopy imaging, they uncovered very interesting temporal and structural aspects of lung epithelial (Club cells) regeneration following toxin exposure. Mostaco-Guidolin et al. (2021) used 3D high-resolution confocal second harmonic generation imaging to investigate and compare collagen fiber organization in alveolar ducts from cleared healthy versus emphysematous mouse lungs. Using detailed image analysis, they found a loss of fibrillary collagen fibers, together with a disruption in the collagen organization in alveolar duct regions from the emphysematous animals.

Bruno and Anathy (2021) provide an overview of the potential use of 3D imaging techniques, such as confocal microscopy and electron microscopy, to investigate the roles epithelial cell endoplasmic reticulum and mitochondria, and their interactions, may play in various lung diseases, including asthma, fibrosis, and infections. Cunniff et al. (2021) provide a brief "Perspective" on how the development of lung-derived organoids, and their 3D imaging may serve as a robust model for studying lung function and alterations in disease. For instance, high-throughput 3D imaging of cultured lung-derived organoids could be used to assess the effects of pharmacological agents on cancerous lung tissue. Finally, in this era of rapidly evolving machine learning image analysis routines, Masquelin et al. (2021) present an alternative convoluted neural network algorithm for screening computed tomography images of human lungs for identification of suspicious lung nodules. They describe the use of a discrete wavelet decomposition (DWT), which compared to the conventional convolutional neural network $(\mathrm{CNN})$ requires the estimation of fewer parameters, reducing the potential of overfitting.

As mentioned in the beginning of this editorial, the whole field of imaging-based pulmonary research would not be the same today without the pioneering work of Prof. Ewald Weibel who passed away on February 19, 2019, shortly before his 90th birthday. Many of the authors who contributed to this SI including ourselves had the luck to know him, discuss with him and to learn from him. Ewald Weibel kept up-todate with the lung imaging field until his very last days and, for sure, he would have loved the idea of a special issue on 3D imaging of the lung. Therefore, as an expression of our respect and gratitude toward him, we would like to dedicate this special issue to him.

We hope you enjoy reading through this Special Issue, and that the diverse manuscripts will provide an appreciation and a representative cross-section of the state-of-the-art techniques in 3D imaging and analysis currently used in lung biology.

\section{References}

Albers J, Pacilé S, Markus MA, Wiart M, Vande Velde G, Tromba G, Dullin C (2018) X-ray-based 3D virtual histology-adding the next dimension to histological analysis. Mol Imaging Biol 20:732-741. https://doi.org/10.1007/s11307-018-1246-3

Baumeister W, Grimm R, Walz J (1999) Electron tomography of molecules and cells. Trends Cell Biol 9:81-85. https://doi.org/10.1016/ s0962-8924(98)01423-8

Borisova E, Lovric G, Miettinen A et al (2021) Micrometer-resolution X-ray tomographic full-volume reconstruction of an intact post-mortem juvenile rat lung. Histochem Cell Biol. https://doi. org/10.1007/s00418-020-01868-8

Briggman KL, Bock DD (2012) Volume electron microscopy for neuronal circuit reconstruction. Curr Opin Neurobiol 22(1):154-161. https://doi.org/10.1016/j.conb.2011.10.022

Bruno SR, Anathy V (2021) Lung epithelial endoplasmic reticulum and mitochondrial 3D ultrastructure: a new frontier in lung diseases. Histochem Cell Biol.

Cox IJ, Sheppard CJ, Wilson T (1982) Improvement in resolution by nearly confocal microscopy. Appl Opt 21:778-781. https://doi. org/10.1364/AO.21.000778 
Cunniff B, Druso JE, van der Velden JL (2021) Lung organoids: Advances in generation and 3D imaging. Histochem Cell Biol. https://doi.org/10.1007/s00418-020-01955-w

Dyba M, Hell SW (2003) Photostability of a fluorescent marker under pulsed excited-state depletion through stimulated emission. Appl Opt 42(25):5123-5129. https://doi.org/10.1364/ao.42.005123

Gehr P, Bachofen M, Weibel ER (1978) The normal human lung: ultrastructure and morphometric estimation of diffusion capacity. Respir Physiol 32:121-140. https://doi.org/10.1016/00345687(78)90104-4

Gil J, Weibel ER (1969/1970) Improvements in demonstration of lining layer of lung alveoli by electron microscopy. Respir Physiol 8:13-36. https://doi.org/10.1016/0034-5687(69)90042-5.

Grothausmann R, Labode J, Hernandez-Cerdan P, Haberthür D, Hlushchuk R, Lobachev O, Brandenberger C, Gie AG, Salaerts T, Toelen J, Wagner WL, Mühlfeld C (2021) Combination of $\mu \mathrm{CT}$ and light microscopy for generation-specific stereological analysis of pulmonary arterial branches: a proof-of-concept study. Histochem Cell Biol. https://doi.org/10.1007/s00418-020-01946-x

Gundersen HJ (1986) Stereology of arbitrary particles. A review of unbiased number and size estimators and the presentation of some new ones, in memory of William R. Thompson J Microsc 143:3-45

Gundersen HJ, Bagger P, Bendtsen TF, Evans SM, Korbo L, Marcussen N, Møller A, Nielsen K, Nyengaard JR, Pakkenberg B et al (1988) The new stereological tools: disector, fractionator, nucleator and point sampled intercepts and their use in pathological research and diagnosis. APMIS 96:857-881. https://doi. org/10.1111/j.1699-0463.1988.tb00954.x

Hsia CC, Hyde DM, Ochs M, Weibel ER; ATS/ERS Joint Task Force on Quantitative Assessment of Lung Structure (2010) An official research policy statement of the American Thoracic Society/European Respiratory Society: standards for quantitative assessment of lung structure. Am J Respir Crit Care Med 181:394-418. https ://doi.org/10.1164/rccm.200809-1522ST

Knudsen L, Brandenberger C, Ochs M (2021) Stereology as the 3D tool to quantitate lung architecture. Histochem Cell Biol. https:// doi.org/10.1007/s00418-020-01927-0

Krischer J-M, Albert K, Pfaffenroth A, Lopez-Rodriguez E, Ruppert C, Smith BJ, Knudsen L (2021) Mechanical ventilation-induced alterations of intracellular surfactant pool and blood-gas barrier in healthy pre-injured lungs. Histochem Cell Biol. https://doi. org/10.1007/s00418-020-01938-x

Low FN (1953) The pulmonary alveolar epithelium of laboratory animals and man. Anat Rec 117:241-264

Maina JN, West JB (2005) Thin and strong! The bioengineering dilemma in the structural and functional design of the blood-gas barrier. Physiol Rev 85:811-844. https://doi.org/10.1152/physr ev.00022.2004

Masquelin AH, Cheney N, Kinsey CM, Bates JHT (2021) Wavelet decomposition facilitates training on small data sets for medical image classifications by deep learning. Histochem Cell Biol. https ://doi.org/10.1007/s00418-020-01961-y

McEwen BF, Radermacher M, Rieder CL, Frank J (1986) Tomographic three-dimensional reconstruction of cilia ultrastructure from thick sections. Proc Natl Acad Sci USA 83:9040-9044. https://doi. org/10.1073/pnas.83.23.9040

Mendis-Handagama SM (1992) Estimation error of Leydig cell numbers in atrophied rat testes due to the assumption of spherical nuclei. J Microsc 168:25-32. https://doi. org/10.1111/j.1365-2818.1992.tb03247.x

Mostaco-Guidolin LB, Loube J, Barlow A, Osei ET, Vasilescu DM, Hsieh A, Fouadi M, Young C, Scott AL, Mitzner W, Hackett TL (2021) Second harmonic generation imaging of collagen scaffolds within the alveolar ducts of healthy and emphysematous mouse lungs. Histochem Cell Biol. https://doi.org/10.1007/s0041 8-020-01959-6
Mühlfeld C, Wrede C, Molnar V, Rajces A, Brandenberger C (2021) The plate body: 3D ultrastructure of a facultative organelle of alveolar epithelial type II cells involved in SP-A trafficking. Histochem Cell Biol. https://doi.org/10.1007/s00418-020-01912-7

Ochs M, Mühlfeld C (2013) Quantitative microscopy of the lung: a problem-based approach. Part 1: basic principles of lung stereology. Am J Physiol Lung Cell Mol Physiol 305:L15-22. https://doi. org/10.1152/ajplung.00429.2012

Ochs M, Nyengaard JR, Jung A, Knudsen L, Voigt M, Wahlers T, Richter J, Gundersen HJ (2004) The number of alveoli in the human lung. Am J Respir Crit Care Med 169:120-124. https:// doi.org/10.1164/rccm.200308-1107OC

Ochs M, Knudsen L, Hegermann J, Wrede C, Grothausmann R, Mühlfeld C (2016) Using electron microscopes to look into the lung. Histochem Cell Biol 146:695-707. https://doi.org/10.1007/s0041 8-016-1502-z

Perez-Bravo D, Myti D, Mizikova I, Pfeffer T, Surat Solaigue DE, Nardiello C, Vadasz I, Herold S, Seeger W, Ahlbrecht K, Morty RE (2021) A comparison of airway pressures for inflation fixation of developing mouse lungs for stereological analyses. Histochem Cell Biol. https://doi.org/10.1007/s00418-020-01951-0

Rawson SD, Maksimcuka J, Withers PJ, Cartmell SH (2020) X-ray computed tomography in life sciences. BMC Biol 18:21. https:// doi.org/10.1186/s12915-020-0753-2

Salwig I, Spitznagel B, Wiesnet M, Braun T (2021) Imaging lung regeneration by light sheet microscopy. Histochem Cell Biol. https ://doi.org/10.1007/s00418-020-01903-8

Schermelleh L, Ferrand A, Huser T, Eggeling C, Sauer M, Biehlmaier O, Drummen GPC (2019) Super-resolution microscopy demystified. Nat Cell Biol 21:72-84. https://doi.org/10.1038/s4155 6-018-0251-8

Schittny JC (2018) How high resolution 3-dimensional imaging changes our understanding of postnatal lung development. Histochem Cell Biol 150:677-691. https://doi.org/10.1007/s0041 8-018-1749-7

Schneider JP, Hegermann J, Wrede C (2021) Volume electron microscopy: analyzing the lung. Histochem Cell Biol. https://doi. org/10.1007/s00418-020-01916-3

Shotton D, White N (1989) Confocal scanning microscopy: threedimensional biological imaging. Trends Biochem Sci 14:435-439. https://doi.org/10.1016/0968-0004(89)90096-0

Sterio DC (1984) The unbiased estimation of number and sizes or arbitrary particles using the disector. J Microsc 134:127-136

Taatjes DJ, Palmer CJ, Pantano C, Hoffmann SB, Cummins A, Mossman BT (2001) Laser-based microscopic approaches: application to cell signaling in environmental lung disease. Biotechniques 31:880-894. https://doi.org/10.2144/01314rv01

Weibel ER (1963) Morphometry of the human lung. Academic Press, New York

Weibel ER (1971) The mystery of "non-nucleated plates" in the alveolar epithelium of the lung explained. Acta Anat 78:425-443. https ://doi.org/10.1159/000143605

Weibel ER, Gil J (1968) Electron microscopic demonstration of an extracellular duplex lining layer of alveoli. Respir Physiol 4:4257. https://doi.org/10.1016/0034-5687(68)90006-6

Willführ A, Brandenberger C, Piatkowski T, Grothausmann R, Nyengaard JR, Ochs M, Mühlfeld C (2015) Estimation of the number of alveolar capillaries by the Euler number (Euler-Poincaré characteristic). Am J Physiol Lung Cell Mol Physiol 309:L1286-L1293. https://doi.org/10.1152/ajplung.00410.2014

Publisher's Note Springer Nature remains neutral with regard to jurisdictional claims in published maps and institutional affiliations. 